\title{
AN EMPIRICAL INVESTIGATION OF THE PSYCHOSOMATOGENIC FAMILY MODEL*
}

\author{
THOMAS W. BuRBECK $\ddagger$
}

(Received 5 July 1979)

\begin{abstract}
This study evaluated the psychosomatogenic family model as an etiological theory of psychosomatic symptomatology among chronically ill asthmatic children. The model postulates that four family transactional characteristics-enmeshment, overprotectiveness, lack of conflict resolution, and rigidity -nurture psychosomatic symptoms among chronically ill children. These four characteristics were measured in the families of 42 asthmatic children, and the severity of each child's asthmatic condition was assessed through parents' ratings, medical records, and physicians' clinical judgements. Factor analysis was used to create composite variables of severity, and multiple regression analysis was used to assess the relationships between the four family characteristics and six dimensions of severity of the asthmatic condition. No evidence was found to support the psychosomatogenic family model, and an inverse relationship was discovered between the four family characteristics and parents' views of severity. Implications of these findings are discussed along with the importance of multidimensional ratings of severity in future research on the psychosomatic aspects of childhood asthma.
\end{abstract}

AMONG chronically ill children, exacerbations of somatic symptoms that cannot be explained by medical science are often attributed to emotional causes. As a result, psychological and social phenomena have come to be considered important influences affecting children who develop chronic, severe, relapsing bronchial asthma despite competent pediatric care, a condition Peshkin and Tuft [1] labeled "intractable asthma". One etiological theory of psychosomatic symptoms among chronically ill children postulates that the dynamics of the family social environment are critical factors in initiating and maintaining intractable asthma. The psychosomatogenic family model [2] forms the foundation of this theory by specifying family characteristics which, when combined with the presence of a chronically ill asthmatic child in the family, create substantial social, emotional, and medical problems for the child. The purpose of the investigation reported in this paper was to assess the extent to which one aspect of the psychosomatogenic family model applies to the families of asthmatic children.

The research literature on associations between social-psychological factors and childhood bronchial asthma has been equivocal. Findings reported prior to 1960, according to Dubo et al. [3] are contradictory and conclusions made from these studies were often based on speculation rather than scientific evidence. Conversely, Owen [4] in his review of the literature for the same period, concluded that problems in the mother-child relationship were a recurrent observation in studies dealing with the psychosomatic aspects of severe bronchial asthma. He also cites evidence that asthmatic symptoms occur in response to a variety of specific stimuli, and that somatic responses, including respiratory function, clearly reflect emotional states.

Minuchin $e t$ al. [2] make additional observations concerning the ambiguity of research on childhood psychosomatic illness. They point out that linear causal models have dominated the conceptual approach to studying psychosomatic illness, and have hindered advances in the field. These models

*This research was funded by a grant from the Leahi Foundation, Honolulu, Hawaii, and was conducted as the author's Master's Thesis at the University of Hawaii School of Social Work.

†Doctoral student in Social Work and Sociology, The University of Michigan, Ann Arbor, MI 48103, U.S.A.

¥The author wishes to acknowledge Walter Hudson and Linda Rich for their important contributions to this research. 
describe psychosomatic illness as residing within the sick individual and as being caused unilaterally by specific elements in the individual's physical, psychological, or social environment.

Pinkerton [5] reviewed evidence of important psychological factors which complicate the expected pathophysiology of the asthmatic condition. His research was unique, as pointed out by Minuchin et al. [2], in its focus on the feedback processes between a child and his social environment. Pinkerton discovered a range of tension-producing environments that correlate with impairment in ventilatory function among asthmatic children. These situations range from good-willed and over-protective gestures of parents, to outwardly threatening or rejecting behaviors [5].

Minuchin and his associates at the Philadelphia United Guidance Clinic have worked extensively over the past 10 years with severe cases of psychosomatically ill children. They developed Structural Family Therapy as an open systems approach to treatment in which psychosomatically ill children are helped within the context of the family. Structural Family Therapy is guided by two major assumptions: (1) that certain types of family organization are related to the development of psychosomatic symptoms in children, and (2) that children's psychosomatic symptoms play a major role in maintaining family homeostasis. The child's symptoms are cued and reinforced in the feedback processes between the child and his family, and the family's reactions to the child's symptoms are also cued and reinforced. The psychosomatically ill child eventually develops a central role in family conflict avoidance and other interactional patterns, and is continually reinforced for his role $[2,6]$.

Based on their experience using Structural Family Therapy to treat the entire family when one of the children has psychosomatic symptoms, the Philadelphia group has developed the model of the psychosomatogenic family. Their model postulates that three factors, in conjunction, initiate and maintain the development of psychosomatic illness in children. First, the child is physiologically susceptible to the condition, i.e. he has some chronic illness. Second, the family of the chronically ill child displays four transactional characteristics; enmeshment, overprotectiveness, rigidity, and lack of conflict resolution. Third, the sick child plays a major role in the family's conflict avoidance mechanisms, and this role is an important source of reinforcement for his symptoms [2].

The psychosomatogenic family model was developed as a theory of etiology for childhood psychosomatic illnesses in general, but has been utilized extensively in explanations of severe symptoms of asthmatic children [7]. The study reported in this paper examined the relationship between symptomatology of asthmatic children and the four family transactional characteristics of the psychosomatogenic family model. Definitions of these four characteristics as extracted from Liebman et al. [7] are given below.

\section{Enmeshment}

Family members are overinvolved with and overresponsive to one another. Change efforts made by any member stimulate a chain of events designed to maintain the status quo and prevent change from occurring. Family members intrude upon each other's thought, feelings, activities, and communications. There is little autonomy and privacy for individual family members, and the generational boundaries between parents and children are weak and easily crossed. Interpersonal boundaries which define where one person leaves off and the other begins are also weak, resulting in a confusing of roles.

\section{Overprotectiveness}

The family members have a high degree of concern for each other; nurturing and protective responses are constantly being elicited and supplied. Parental overprotectiveness results in few extrafamilial relationships and activities for the patient. When the patient becomes sick, the entire family becomes organized around his care, often submerging intrafamilial conflicts in the process.

\section{Rigidity}

The family often presents itself as being completely normal without any problems except for the patient's medical problems. Therefore, they deny the need for change within the family system, and they preserve accustomed patterns of interaction and behavior.

\section{Lack of confict and resolution}

There is a low tolerance for overt conflict in these families. Confrontations involving differences of opinion and issues of autonomy and control are avoided or diffused. Consequently, there is a chronic state of submerged, unresolved conflict, with associated stress and tension. The child with severe psychosomatic symptoms plays a vital part in the family's avoidance conflict. The experience of being able to protect the family from conflict, especially the parents, by way of symptoms is a powerful reinforcement to the patient. In addition, the sibling subsystem may reinforce the symptoms as part of a protective and/or a scapegoating system. 
In the psychosomatogenic family, these four characteristics eventually dominate the interactions of the members, and the child's illness becomes an important conflict avoidance mechanism. The model defines the four elements as pathological exclusively for families with chronically ill children, making no assumptions about the consequences of these characteristics when they exist in other families.

In a recent review of 75 research studies concerning the effectiveness of marital and family therapies, Structural Family Therapy applied to the problems of psychosomatically ill children was heralded as offering "clearly the most impressive results" [8]. The Philadelphia group reported their results of 10 successfully treated intractable asthma cases in the literature reviewed for this paper, and they cite these findings as evidence of the validity of the psychosomatogenic family model $[2,7,9]$.

\section{SUBJECTS AND METHOD}

The purpose of the study reported in this paper was to examine, through survey research methods, the validity of the psychosomatogenic family model. As described, this model postulates that four specific family transactional characteristics are central factors in the maintenance of psychosomatic symptoms among chronically ill children. A sample of asthmatic children and their families was selected, and a set of predictor variables was defined as the extent to which each family displayed the transactional characteristics posited in the psychosomatogenic family mode. Criterion variables were constructed from a variety of measures of the severity of each child's asthmatic condition. It was hypothesized that the severity of an asthmatic child's condition correlates positively with the extent to which his family demonstrates transactional patterns that conform to the psychosomatogenic family model.

Forty-two asthmatic children, roughly half males and half females, and the primary care-giving parent or guardian of each provided data for the study. Eighty-three per cent of this sample was obtained from the pediatric pulmonary service of a children's hospital in Hawaii, U.S.A. These thirty-five cases represented about $90 \%$ of the service's asthma patients who were appropriate for the study (i.e. under $20 \mathrm{yr}$ of age and living in a family setting). Seventeen per cent of the sample was obtained from an allergy physician's private practice in Hawaii.

The forty-two asthmatic children surveyed had a mean age of 10 , ranging from 2 to 19 . Sixty-two per cent of the children were from mixed ethnic backgrounds, a characteristic common to the people residing in Hawaii. Twelve per cent were Filipino, $10 \%$ were Caucasian and $7 \%$ were Japanese. Eighty-eight per cent of the adults interviewed for the study (i.e. the primary care-giving parents and guardians) were the natural mothers of the asthmatic children.

The major portion of the data was collected through an interview with each primary care-giving parent or guardian conducted by one of three trained interviewers. One series of interview questions elicited the respondent's assessment of observable behaviors his asthmatic child displayed in response to the medical condition. This involved ratings of the following: frequency and duration of wheezing and shortness of breath; number of school days missed due to asthma; frequency of hospital emergency room and asthma clinic visits due to asthma; and admissions to, and days spent in, the hospital due to asthma.

Each interview respondent completed an Index of Family Characteristics (IFC) to provide a second source of data. The IFC was designed to assess the extent to which a family displays the transactional characteristics postulated in the psychosomatogenic family model. Descriptions of the psychosomatogenic family published by the Philadelphia group $[2,6,9,10]$ were carefully assessed, and specific family behaviors were thought of that could fit the descriptions. The IFC was developed by stating these behaviors in a form that could be rated by a family member in terms of how frequently the behavior occurs in his family. For example, "When something happens to one person in my family, the other members find out about it" is rated by the respondent on a six point Likert scale ranging from "Almost Never" to "Almost Always". Four such items were developed in this way to represent each of the four transactional characteristics of the psychosomatogenic family mode. The IFC was used to rate the family of each asthmatic child on a 100 point scale for each of the four family characteristics, a score of 100 representing maximum presence of the characteristic.

Medical information recorded throughout the previous year was obtained for each asthmatic child by examining his medical charts. A form was created for systematically recording each subject's number of hospital emergency room visits due to asthma, number of admissions to, and days spent in, the hospital due to asthma, and number of visits to an asthma clinic.

A third method of data collection utilized the medical ratings of two physicians working regularly with the asthmatic children in the sample. Through consultations with these doctors, rating scales were devised so that each doctor could systematically report on five characteristics of each patient's asthma-related behavior and medical regimen. These ratings included chronicity of the medical condition, adherence to the prescribed medication, and stability of the asthmatic condition. A fourth measure, level of medication, was adapted from the earlier work of Weinberger [11] and was also 
used by the physicians to rate each child. Examples of the items of all the data collection instruments used for the study are displayed in the Appendix.

\section{DATA ANALYSIS}

Multiple regression analysis provided the best means for describing the data and testing the explicit hypothesis. Six composites comprised of various measures of each child's behavior due to his asthmatic condition were used as criterion variables. The four dimensions measured by the IFCenmeshment, overprotectiveness, lack of conflict resolution, and rigidity-were defined as predictor variables in a series of multiple regression models.

The Index of Family of Family Characteristics was developed exclusively for this study and its reliability was carefully examined before proceeding with the formal analyses. Coefficient Alpha [12] was used to estimate reliability for each subscale, and the results of this analysis are presented in Table 1. Descriptive statistics for each subscale are presented in Table 2.

Table 1.-Reliability analysis on IFC subscales

\begin{tabular}{lcr}
\hline Subscale name & Item $N$ & Alpha \\
\hline Enmeshment & 4 & $\mathbf{0 . 3 3 6}$ \\
Overprotectiveness & 4 & 0.574 \\
Rigidity & 4 & 0.545 \\
Lack of conflict resolution & 4 & $\mathbf{0 . 6 0 3}$ \\
\hline
\end{tabular}

TABLE 2.-DESCRIPTIVE STATISTICS FOR IFC subscales

\begin{tabular}{lccc}
\hline Subscale name & Mean & $\begin{array}{l}\text { Standard } \\
\text { deviation }\end{array}$ & Skewness \\
\hline Enmeshment & 64.083 & 18.136 & 0.099 \\
Overprotectiveness & 58.542 & 14.203 & 0.073 \\
Rigidity & 47.917 & 13.428 & $-\mathbf{0 . 1 5 3}$ \\
Lack of conflict resolution & 49.625 & 16.656 & $\mathbf{0 . 1 4 8}$ \\
\hline
\end{tabular}

It is very important to note that the subscale reliability coefficients were quite low, especially in the case of the enmeshment subscale. Because the upper limit on scale validity is determined by its reliability [13], the validity of the enmeshment subscale scores was open to serious question. Low reliability in a predictor variable makes estimates using that variable conservative. Thus, by using the enmeshment subscale scores in the final analysis, the probability of Type II errors was increased.

The criterion variables were selected to reflect the severity of the children's observable and quantifable behaviors resulting from their asthmatic conditions. Seventeen interview, medical record, and physician rating scale items were used to gather information on the severity of each child's asthmatic condition. A principle components analysis was conducted on these seventeen items to reduce the data to a more manageable form while retaining as much of its information as possible. Collapsing the items in this manner allowed a maximum amount of information to be retained for the final analyses while not maintaining a high ratio of variables to sample size.

An initial principle components analysis in which the computed factors were allowed to be correlated with each other (oblique rotation) indicated that inter-factor correlations were small enough to permit an orthogonal rotation without sacrificing either an unacceptable amount of explained variance in the original seventeen severity variables or the conceptual logic of the severity factor composites. Thus a second principle components analysis using orthogonal (Varimax) rotation was conducted and six uncorrelated factors were extracted from the original seventeen variables. Table 3 presents the seventeen variables which comprised the severity factors, along with their factor loadings. Each variable with a factor loading of 0.40 or greater was included in the naming of that severity factor. Table 4 presents the factor names and the set of variables loading heavily on each.

The first factor, 'Hospital Inpatient Time', was made up almost exclusively of the four variables showing how often and for how long each child had been in the hospital due to his problems with asthma. It is important to note that short term contacts with medical facilities, e.g. emergency room visits, load only lightly on this factor.

'Parent's View of Severity', the second factor, appeared to represent the parents' and guardians' overall assessments of the problems the children had with asthma. All items loading substantially on this factor were parent ratings which suggests the importance of their view as distinct from medical judgements and measures obtained from medical records. 
TABLE 3.-FACTOR LOADINGS OF SEVERITY VARIABLES ON SIX SEVERITY FACTORS"

\begin{tabular}{lrrrrrr}
\hline Variable name & Factor 1 & Factor 2 & Factor 3 & Factor 4 & Factor 5 & Factor 6 \\
\hline Wheezing frequency (P) & 0.1958 & $0.4041 \dagger$ & $0.7388 \dagger$ & 0.1488 & 0.1978 & -0.1973 \\
Short breath frequency (P) & -0.0911 & 0.1481 & $0.8487 \dagger$ & 0.2744 & 0.0357 & -0.0650 \\
Wheezing duration (P) & -0.1249 & -0.1890 & 0.1257 & -0.0914 & -0.2048 & $0.8811 \dagger$ \\
Short breath duration (P) & 0.0728 & $0.4444 \dagger$ & -0.1178 & 0.0703 & 0.1832 & $0.7813 \dagger$ \\
School days missed (D) & -0.2166 & $0.8276 \dagger$ & 0.2150 & 0.1705 & 0.1731 & 0.1245 \\
Emergency room visits (P) & -0.0242 & -0.0313 & $0.7803 \dagger$ & 0.2095 & 0.2273 & 0.2607 \\
Asthma clinic visits (P) & 0.1041 & $0.8103 \dagger$ & 0.1105 & 0.0005 & -0.0433 & 0.0210 \\
Hospital admissions (P) & $0.7702 \dagger$ & $0.5688 \dagger$ & 0.2083 & 0.0795 & -0.0142 & 0.0087 \\
Days in hospital (P) & $0.4646 \dagger$ & $0.6664 \dagger$ & 0.0327 & 0.1850 & 0.0378 & -0.0973 \\
Emergency room visits (R) & 0.2399 & 0.1719 & $0.4366 \dagger$ & -0.0184 & $0.5320 \dagger$ & 0.0590 \\
Hospital admissions (R) & $0.9721 \dagger$ & -0.0337 & -0.0168 & 0.1535 & 0.0403 & -0.0330 \\
Days in hospital (R) & $0.9333 \dagger$ & 0.0073 & -0.0408 & 0.2075 & 0.0190 & -0.0352 \\
Asthma clinic visits (R) & -0.0146 & 0.3805 & -0.0644 & 0.2158 & $0.6960 \dagger$ & -0.2789 \\
Chronicity (D) & 0.2670 & 0.0560 & 0.2730 & $0.8333 \dagger$ & 0.0223 & 0.0010 \\
Regimen cooperation (D) & -0.0692 & -0.1910 & 0.3210 & -0.0277 & $0.7096 \dagger$ & 0.0570 \\
Instability of condition (D) & 0.2598 & 0.0266 & 0.0978 & $0.7142 \dagger$ & 0.3759 & 0.0501 \\
Intensity of medication (D) & 0.0297 & 0.1733 & 0.1650 & $0.7507 \dagger$ & -0.1008 & -0.0723 \\
\hline
\end{tabular}

*For each variable name '(P)' indicates rating by parent or guardian, '(R)' indicates ratings from medical records, and '(D)' indicates rating by the doctor.

†Factor loadings over 0.40 were used in naming respective factors.

TABLE 4.-SEVERTYY FACTOR DEFINITIONS

\begin{tabular}{lc}
\hline Factor definitions & Factor weights \\
\hline FACTOR 1: HOSPITAL INPATIENT TIME & \\
Frequency of hospital admissions (R) & 0.972 \\
Days spent in hospital (R) & 0.933 \\
Frequency of hospital admissions (P) & 0.770 \\
Days spent in hospital (P) & 0.465 \\
FACTOR 2: PARENT'S VIEW OF SEVERITY & \\
Days of school missed (P) & 0.828 \\
Frequency of clinic visits (P) & 0.810 \\
Days spent in hospital (P) & 0.666 \\
Frequency of hospital admissions (P) & 0.569 \\
Duration of shortness of breath (P) & 0.444 \\
Frequency of wheezing (P) & 0.404 \\
FACTOR 3: EPISODE FREQUENCY AND INTENSITY & \\
Frequency of shortness of breath (P) & 0.849 \\
Frequency of hospital emergency room visits (P) & 0.780 \\
Frequency of wheezing (P) & 0.739 \\
Frequency of hospital emergency room visits (R) & 0.437 \\
FACTOR 4: DOCTOR'S VIEW OF SFVERITY & \\
Chronicity of asthmatic condition (D) & $\mathbf{0 . 8 3 3}$ \\
Strength of prescribed medication (D) & 0.751 \\
Instability of medical condition (D) & $\mathbf{0 . 7 1 4}$ \\
FACTOR 5: RESISTANCE TO MEDICAL REGIMEN & \\
Failure to cooperate with doctor (D) & 0.710 \\
Frequency of clinic visits (R) & $\mathbf{0 . 6 9 6}$ \\
Frequency of hospital emergency room visits (R) & 0.532 \\
FACTOR 6: EPISODE DURATION & \\
Duration of wheezing (P) & 0.881 \\
Duration of shortness of breath (P) & 0.781 \\
\hline
\end{tabular}

'Episode Frequency and Intensity', as the third factor, quite clearly represented how often the asthma flared up and how often these episodes were severe enough to require emergency medical attention. This factor was considered highly indicative of short term, potentially life-threatening asthma attacks. 
Factor Four, 'Doctor's View of Severity', was dominated by the physicians' ratings of chronicity, strength of medication, and instability of condition. It was defined as the professional medical assessment of severity.

'Resistance to Medical Regimen', Factor Five, was thought to reflect the extent of failure of the patients to adhere to the doctors' orders, primarily concerning prescribed medicine. The second and third variables loading substantially on this factor, clinic visits and emergency room visits, were thought to represent two results of improper home treatment of the asthmatic condition.

'Episode Duration', the last factor, quite simply indicated how long each episode of asthma symptoms lasted. The close similarity of the two items loading heavily on this factor contributed markedly to its conceptual cohesiveness.

The primary hypothesis which guided the analysis was, as previously stated, that children's asthma problems are positively associated with high levels of family enmeshment, overprotectiveness, lack of conflict resolution, and rigidity. The mathematical models used to test this hypothesis consisted of six univariate multiple regression equations using four predictor variables and six criterion variables.

The six computed severity factors of each child's behavioral response to his asthma were treated as criterion variables. Because of the orthogonal rotation used in the principal components analysis each factor was uncorrelated with the other five factors. This independence among factors allowed each to be used as a criterion variable in a separate multiple regression model without contamination due to non-independence of the regression $F$ tests. Thus, each criterion variable was predicted in each equation using the least-squares, best-fitting linear combination of the four IFC subscale scores.

In testing each model, all four predictor variables were entered into the equation on a single step to test their simultaneous contribution to explained variance in each criterion variable. Due to the small sample size, the low number of degrees of freedom in each model, and the low Short-Form IFC subscale reliabilities, the confidence level for the $F$ statistic for each model was set at $p<0.10$.

\section{FINDINGS}

The data collected for this study, when analysed according to the six mathematical models described above, provided no evidence to support the research hypothesis. The $F$ statistic in each case indicated no significant positive relationship at the $p<0.10$ confidence level between severity of asthmatic condition and extent of family enmeshment, overprotectiveness, lack of conflict resolution, and rigidity. Summary statistics for the tests of these six models are reported in Table 5.

TABLE 5.-SUMMARY STATISTICS FOR TESTS OF SIX a priori MODELS

\begin{tabular}{lcccc}
\hline MODEL: Criterion variable* & $\begin{array}{c}\text { Multiple } \\
K\end{array}$ & $\begin{array}{c}F \\
\text { statistic }\end{array}$ & $\begin{array}{c}\text { Degrees of } \\
\text { freedom }\end{array}$ & probability \\
\hline MODEL ONE: Hospital inpatient time & 0.415 & 1.719 & $4-33$ & $p>0.10$ \\
MODEL TWO: Parent's view of severity & 0.524 & 3.128 & $4-33$ & $p<0.05 \dagger$ \\
MODEL THREE: Episode frequency and intensity & 0.272 & 0.661 & $4-33$ & $p>0.10$ \\
MODEL FOUR: Doctor's view of severity & 0.403 & 1.599 & $4-33$ & $p>0.10$ \\
MODEL FIVE: Resistance to medical regimen & 0.412 & 1.685 & $4-33$ & $p>0.10$ \\
MODEL SIX: Episode duration & 0.406 & 1.631 & $4-33$ & $p>0.10$ \\
\hline
\end{tabular}

*The predictor variable set for each model was composed of the four Short-Form IFC subscales: enmeshment, overprotectiveness, rigidity, and lack of conflict resolution.

$\dagger$ Although the test of this model was statistically significant at the 0.05 probability level, the relationship was in the negative direction as indicated by a negative sign on the standardized beta weight for each predictor variable. This was opposite the hypothesized direction and its interpretation therefore constituted a post hoc analysis.

It was not the purpose of this study to conduct exploratory, post hoc analyses on the data. However, a pronounced relationship between the criterion and predictor variables in the second model was difficult to overlook, even though it did not support the $a$ priori hypothesis. The $F$ statistic for Model Two indicated that the second behavioral response factor, 'Parent's View of Severity', was negatively related to family enmeshment, overprotectiveness, lack of conflict resolution. and rigidity at the $p>0.05$ confidence level $(F+3.128$; d.f. $+4 / 33)$. In other words, the adults interviewed who reported low levels of the characteristics postulated in the psychosomatogenic family model tended to also report high severity on Factor Two, 'Parent's View of Severity'.

Negative beta weights on each of the predictor variables in Model Two provided clear evidence that this was an inverse relationship. The multiple correlation coefficient indicated a substantial relationship $(R+0.524)$, and when squared showed that almost $30 \%$ of the variance in 'Parent's View of Severity' could be accounted for by variation in the family transaction measures $\left(R_{\mathbf{b}}+\mathbf{0 . 2 7 5}\right)$. 


\section{DISCUSSION}

This study was conducted in search of empirical support for the psychosomatogenic family model. Numerous precautions were taken to (1) develop a survey instrument which could accurately measure the four transactional characteristics of the model, (2) utilize diverse and numerous indicators of severity of the asthmatic condition, (3) obtain a subject sample having a good chance of displaying the dynamics described by the model, and (4) build powerful mathematical models of analysis that would detect as well, as possible any relationships that existed in the data. No evidence was found to support the model's application to the families of asthmatic children used for this study.

The main criticism of the research design might concern the subjects which were surveyed. Liebman et al. [9] cite evidence that children with intractable asthma constitute only $10-12 \%$ of all asthmatic children. The research reported by Sultz [14] indicates that only $55 \%$ of the families with asthmatic children seek on-going medical care for the disease. The other $45 \%$ have contact with medical facilities only for emergency episodes of their children's asthma. By assuming that the families used for this study were representative of that $55 \%$ regularly obtaining pediatric care for their asthmatic children, it was estimated that about $20 \%$ of the families in the sample had children with intractable asthma. (Out of every 100 asthmatic children, 55 have consistent pediatric care, and $10-12 \%$ of these 55 have intractable asthma.) If this were the case for the sample in this study, the $80 \%$ of the children who didn't have intractable asthma might have masked the effects of the more severe $20 \%$.

However, the study examined severity of asthma as a continuous variable in order to test whether the psychosomatogenic family model could differentiate among the various levels of severity. It was necessary to use a range of cases less severe than 'intractable' for this comparison process. The $20 \%$ of the children having the most severe symptoms were expected to be members of those families reporting the highest levels of enmeshment, overprotectiveness, lack of conflict resolution, and rigidity. The model should have also been able to account for the range of milder cases falling below the most severe level by showing lesser amounts of the four transactional characteristics in the families of these children.

A second possible fault of the research design concerns the use of the Index of Family Characteristics as a measure of the four transactional characteristics described by the model. As reported earlier, the IFC subscale reliabilities were quite low, especially in the case of enmeshment. It is highly possible that these low reliabilities can account for the lack of support for the research hypothesis. It is important to note, however, that the post hoc analysis showed a statistically significant negative relationship of a fairly large magnitude $(R=0.524)$ using the four subscales. This lends credibility to the IFC by showing that it could account for a substantial amount of the variation in at least one of the severity factors.

The reliability coefficient on the total IFC scale, before dividing it into subscales, was fairly high (i.e. alpha $=0.79$ ). It was tempting to use the total IFC scores as a single predictor variable in six additional mathematical models to see how it correlates with each of the six severity factors. However an item analysis on the ShortForm IFC indicated that the scale is, in fact, multidimensional. The multiple group factor analysis that was conducted confirmed the $a$ priori subscale structure, i.e. the four subscales did measure four different dimensions. Another factor analysis on the 
IFC items using oblique rotation confirmed this finding of multi-dimensionality, and indicated that using the IFC as a unidimensional scale to predict each of the severity factors would be unacceptable.

Another important point concerning the development of the IFC items cannot go without mention. The scale items were created by simply reading about and trying to understand the psychosomatogenic family model, and then attempting to put the model into quantifiable terms. Because of this, the validity of the Short-Form IFC is open to serious question.

These findings of limited validity on the IFC raise an important issue concerning the research of the Philadelphia group. As Sidman [15] points out, one of the necessities of sound theory development in the behavioral sciences is clear communication between scientists. Without such interaction, it proves difficult for behavioral scientists to replicate research findings and hence build theories. When communication regarding a specific theory is not sufficiently descriptive to develop valid measurement instruments, $i \mathrm{i}$ is reasonable to question the tenacity of the theory itself. Such questioning may be warranted in the case of the psychosomatogenic family model. But it should also be noted that probably the biggest problem faced today in the behavioral sciences concerns quantification of human behavior. Accurately measuring even simple behaviors is often a difficult task. Quantifying the intricacies of family interactions, as is necessary to validate the psychosomatogenic family model, presents a monumental undertaking.

A final criticism of this investigation might concern the size of the sample relative to the types of statistical analyses that were conducted. Each of the models developed to test the research hypothesis utilized composites of other variables and multiple variable equations. The restrictions on the degrees of freedom were quite high because of this, and true relationships had to be fairly large before they reached the specified statistical significance levels.

The findings of this investigation, when combined with the lack of sound, empirical evidence produced by other research, suggest that the psychosomatogenic family model as applied to asthmatic children is of questionable validity. Gurman and Kniskern [8], in their extensive review of research studies on the effectiveness of marital and family therapies, cited only one study concerning Structural Family Therapy that used control groups. That study [16] was unpublished and unavailable at the time of this writing.

An examination of the quality of the research reported by the Philadelphia group on the psychosomatogenic family model and its application to the families of asthmatic children indicated that this research is extremely limited and inconclusive. The Philadelphia group has based their conclusions on an unacceptably small number of cases (ten as of this writing) and all of the published research supporting their theory, as applied to asthmatic children, utilizes single-case, time series designs. While such designs appear to provide valuable ways of monitoring treatment as evidenced by the literature on behavior modification therapy, findings of idiographic research, without the confirmations of nomothetic research, can produce only tenuous support for theories of human behavior. The results presented to validate the psychosomatogenic family model are also based on an extremely select problem population. Liebman et al. [9] estimate that the patients treated by their methods come only from the most severe cases of psychosomatically ill children. Yet the psychosomatogenic family 
model attempts to explain the etiology of all psychosomatic asthma symptoms regardless of severity.

The way a problem is rectified does not automatically demonstrate the way it developed. Ignorance of this fact appears to be a conceptual flaw running throughout the writings on the psychosomatogenic family model. The model postulates cause: When there is an asthmatic child living in a family with certain transactional characteristics, that child's asthma has a greater chance of becoming intractable. Yet all of the evidence on which the model is based concerns the effectiveness of a form of therapy designed around the model (i.e. Structural Family Therapy). The research reviewed for this study provided absolutely no evidence to suggest that families with nonpsychosomatically ill asthmatic children are not highly enmeshed, over protective, lacking in conflict resolution skills and extremely rigid.

One of the most important yet easily overlooked results of this investigation concerned the six severity factors. In the research on psychosomatic symptoms among asthmatic children, some index of asthma-related problems is often utilized. This study used a wide variety of measures to get at the notion of 'severity' of the asthmatic condition, and factor analysed these to find common components. The fact that fairly distinct and conceptually logical factors could be extracted gives clear evidence of the multidimensionality of severity of asthmatic problems. Past research on asthma has been dominated by a notion of unidimensional severity. This finding suggests that future investigations should consider specific dimensions in the behavioral expression of asthma.

This finding of multidimensionality of asthma severity might be able to explain some of the problems of past research in detecting significant relationships. For example, Pinkerton [17] developed a scale of asthma severity made up of three components: school days missed, severity of attacks, and type of medication. This severity scale was used as a major part of the published findings of the Philadelphia group. The evidence produced by the investigation reported in this paper suggests that each of these three symptoms might be better assessed independently. Combining them could mask important relationships, no matter how rigorous and logical the measurement of predictor variables.

The findings reported in this paper are not, by any means, proof that the psychosomatogenic family model is an invalid representation of etiological factors in the severity of childhood asthma. The findings simply fail to provide evidence to support the model, and suggest that the relationship between family characteristics and one view of asthma severity is exactly opposite what the model predicts. As a guideline for treatment of psychosomatic disorders among asthmatic children, the psychosomatogenic family model might be a valuable tool. But future research must be careful not to confuse issues of treatment effectiveness and theories of etiology. To do so creates a substantial hindrance to scientific progress in this area.

\footnotetext{
A. Interview ratings of severity

APPENDIX

1. How frequently does your child have wheezing from asthma? (Constantly; Hourly; Daily; Weekly; Monthly; Less often than monthly)

2. How frequently does your child have shortness of breath from asthma? (Constantly; Hourly; Daily; Weekly; Monthly; Less often than monthly)

3. How long do your child's periods of wheezing from asthma usually last? (Minutes; Hours; Days; Weeks; Not applicable)
} 
4. How long do your child's periods of shortness of breath from asthma usually last? (Minutes; Hours; Days; Weeks; Not applicable)

5. How many days of school did your child miss during the last school year (1976-1977) due to asthma?

6. How many times in the past year did your child go to a hospital emergency room because of asthma?

7. How many times in the past year did your child go to the doctor and/or asthma clinic because of asthma?

8. How many times in the past year was your child admitted to the hospital because of asthma?

9. How many days in the past year has your child spent in the hospital because of asthma?

\section{B. Medical records ratings of severity}

1. Number of hospital emergency room visits due to asthma during 1977.

2. Number of hospital admissions due to asthma during 1977.

3. Number of days spent in the hospital due to asthma during 1977.

4. Number of visits to an asthma clinic during 1977.

\section{Physician's ratings of severity}

1. How often does this patient suffer from symptoms of asthma? (Less than six times per year; About once every two months; About once per month; About once per week; Almost daily)

2. How often does this patient (and/or the person responsible for administering medication) adhere to his/her medical regimen by taking medication as prescribed? (Most or all of the time; A good part of the time; Sometimes; A little of the time; Rarely or none of the time)

3. How stable is this patient's asthma? That is, how fluctuant are his/her behavioral responses to the disease (e.g. ER visits) compared to what you would expect from knowing how other patients respond to a similar regimen? (Stable; Somewhat stable; Unstable)

4. What type(s) of medicine is the patient currently using? Check all that apply. (PRN - as needed; Theophylline; Inhaled sympathomimetic; Chromolyn and/or oral sympathomimetic; Inhaled corticosteroids; Oral corticosteroids)

\section{Index of family characteristics}

(All items were rated on a six point Likert scale: Almost never; Seldom; Occasionally; Often; Very often; Almost Always. Starred items $\left(^{*}\right)$ are reverse scored.)

\section{Enmeshment}

1. When something happens to one person in my family, the other members find out about it.

*2. When two people in my family have an argument, the other family members leave them alone to settle it by themselves.

3. When it comes to personal problems, the members of my family talk with each other about them.

4. My family is a close-knit group.

\section{Overprotectiveness}

1. When someone is sick in the family, everyone tries to make sure he (or she) is comfortable.

*2. The people in my family are very independent.

3. When one family member has a problem, everyone in the family worries about him (or her).

*4. When one of the family members is feeling a little sick, the other members seem unaware of it.

Rigidity

1. My family feels content with the way things are now.

*2. My family is flexible in what it expects from its members.

3. Family customs are very important in my family.

*4. My family likes to find new ways of doing things together.

\section{Lack of confict resolution}

*1. Arguments in my family, once started, go on until things are worked out.

2. My family tries hard to avoid arguments.

*3. After an argument, the members of my family understand each other better.

4. In my family, arguments are cut short. 


\section{REFERENCES}

1. Peshin M. M. and Tuft H. S. Rehabilitation of the intractable asthmatic child by the institutional approach. $Q$. Rev. Pediatrics 11, 7 (1956).

2. Minuchin S., Baker L., Rosman B. L., Liebman R., Milman L. and Todd T. C. A conceptual model of psychosomatic illness in children. Archs Gen. Psychiat. 32, 1031 (1975).

3. Dubo S., Mclean J. A., Ching A. Y. T., Wright H., Kaufman P. E. and Sheldon J. M. A Study of relationships between family situation, bronchial asthma, and personal adjustment in children. J. Pediatrics 59, 402 (1961).

4. OWEN F. W. Patterns of respiratory disturbance in asthmatic children evoked by the stimulus of the mother's voice. Acta Psychother. 11, 228 (1963).

5. PINkerton P. Pathophysiology and psychopathology as co-determinants of pharmacotherapeutic response in childhood asthma. Psychotropic Drugs in Internal Medicine (Edited by PleTsCher A. and Marino A.), p. 115. Excerpta Medica Foundation, Amsterdam (1969).

6. Minuchin S. Families and Family Therapy. Harvard Press, Cambridge, Mass. (1974).

7. Liebman R., Minuchin S., Baker L. and Rosman B. L. The role of the family in the treatment of chronic asthma. Family Therapy Theory and Practice (Edited by GUERIN T. J.). p. 309. Gardner Press, New York (1976).

8. GuRMan A. S. and KNISKern D.P. Research on marital and family therapy: progress, perspective and prospect. Handbook of Psychotherapy and Behavior Change, 2nd Edn. (Edited by GARFIELD S. L. and Bergin A. E.). Wiley, New York (in press).

9. Lierman R., Minuchin S. and BakfR L. The use of Structural Family Therapy in the treatment of intractable asthma. Am. J. Psychiat. 135, 535 (1974).

10. Liebman R., Minuchin S. and BAKER L. An integrated treatment program for anorexia nervosa. Am. J. Psychiat. 131, 432 (1974).

11. WeInberger M. Guidelines on uptimal safe usage of oral theophylline for asthma, chronic bronchitis, and emphysema. Ad. Asthma, Allergy and Pulmonary Dis. 4, 25 (1977).

12. Nunnally J. C. Psychometric Theory. McGraw-Hill, New York (1967).

13. Helmstadter G. C. Principles of Psychological Measurement. Prentice-Hall, Englewood Cliffs, N. J. (1964).

14. Sultz H. A. Long-term childhood illness. Contemporary Community Health, University of Pittsburgh Press, Pittsburgh (1972).

15. Sidman M. Tactics of Scientific Research. Basic Books, New York (1960).

16. Stanton M. D. and Todd T. C. Structural family therapy with heroin addicts: some outcome data. Paper presented at the Society of Psychotherapy Research meeting, San Diego, California (June 1976).

17. Pinkerton P. and Weaver C. M. Childhood asthma. Modern Trends in Psychosomatic Medicine (Edited by Hill O.), p. 81. Butterworths, London (1970). 